\title{
PR@SPECT
}

\section{Limit on sub-GeV dark matter from PROSPECT experiment}

Manoa Andriamirado (IIT)

On behalf of the PROSPECh $\bar{F}_{\bar{v}}$ Collaboration

Phys. Rev. D 104, 012009 


\section{Dark-Matter direct detection}

- DM direct detection focuses on GeV-scale.

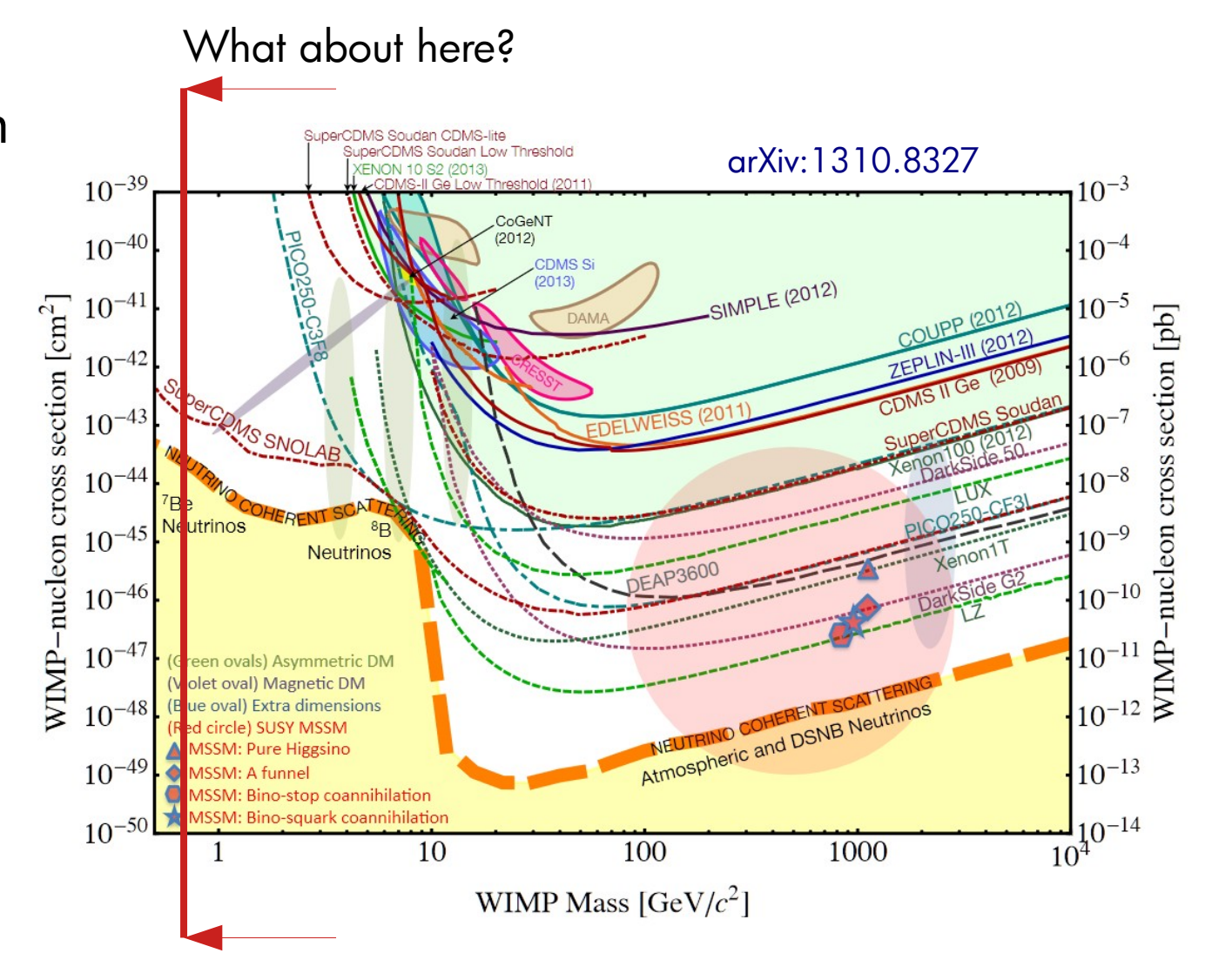

- Light DM does not have enough momentum to trigger detector.

- DM can gain energy through DM-cosmic rays scattering.

- In higher cross-section space, overburden will actively block DM signals!

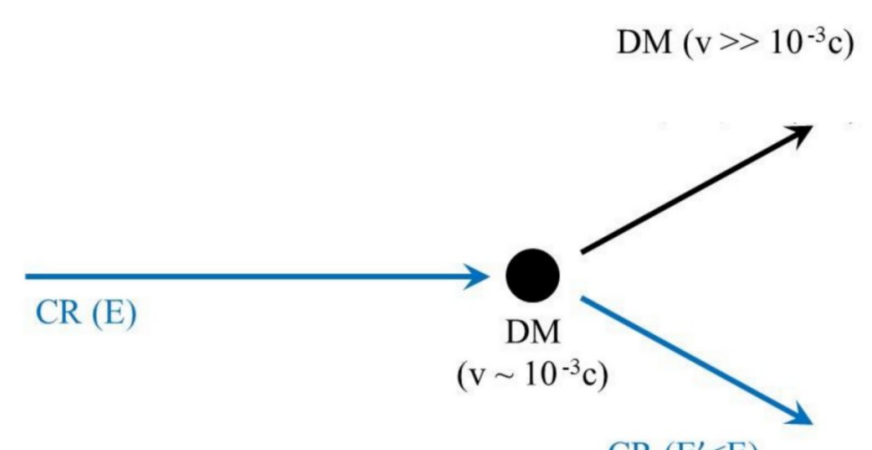




\section{Dark-Matter propagation}

- Assume Navarro-Frenk-White DM profile.

- Cosmic-rays halo is modeled as a cylinder with $\mathrm{R}=25 \mathrm{kpc}$ and $\mathrm{h}=4 \mathrm{kpc}$.

- Use Local Interstellar spectrum for the CR energy distribution.

- Propagate through atmosphere and detector shielding.

- DM flux in the detector vary as the earth rotate:

- High signal period

- Low signal period

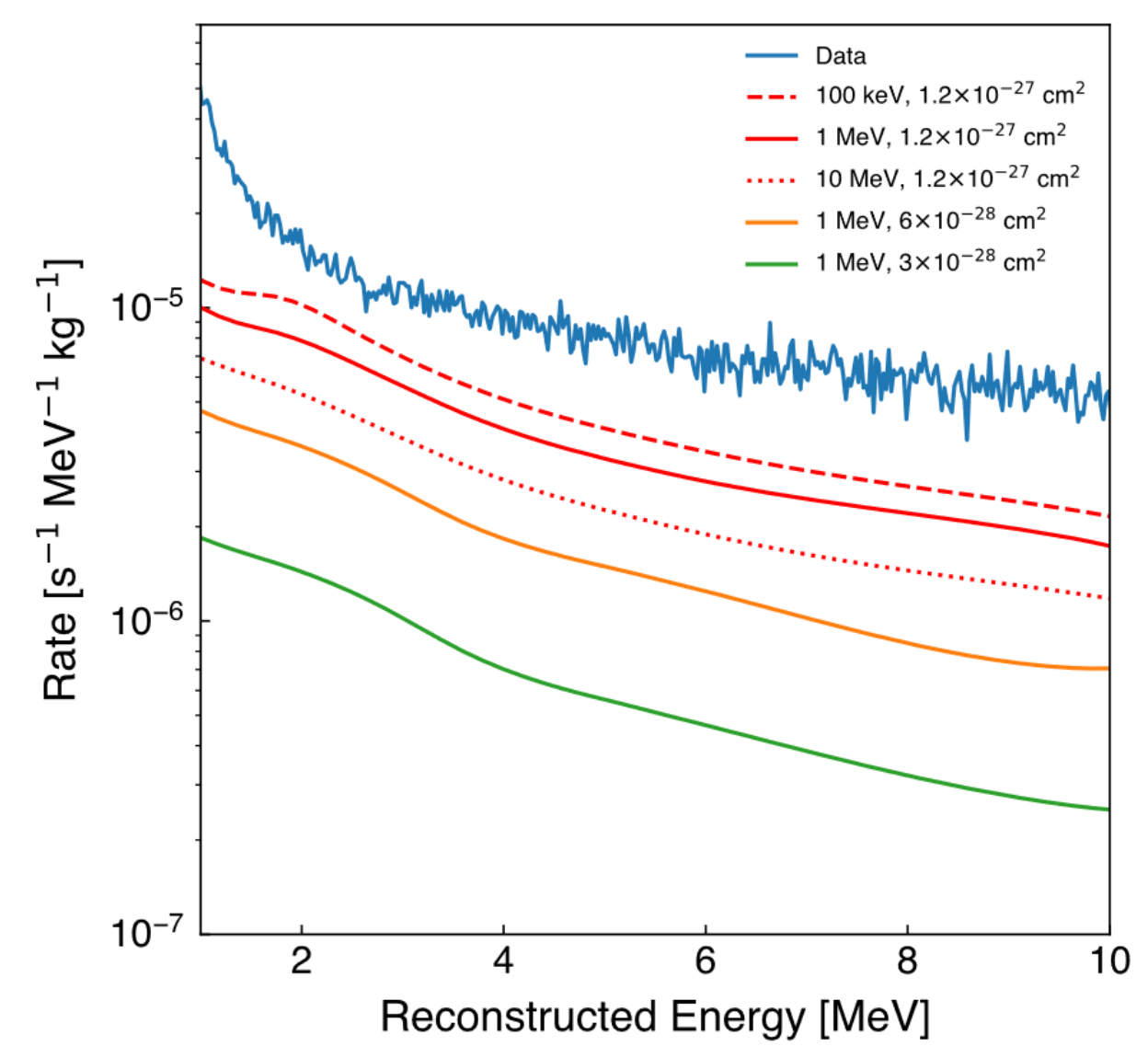




\section{The PROSPECT Experiment \& Detector}

- Designed to measure ${ }^{235} \mathrm{U}$ spectrum and search for sterile neutrino oscillations.

- Deployed on surface with minimal overburden by the High Flux Isotope Reactor at Oak Ridge National Laboratory.

- The detector consists of $11 \times 14$ optically isolated segments filled with ${ }^{6}$ Li-doped liquid scintillator (LiLS).

- Double ended PMTs for each segment.

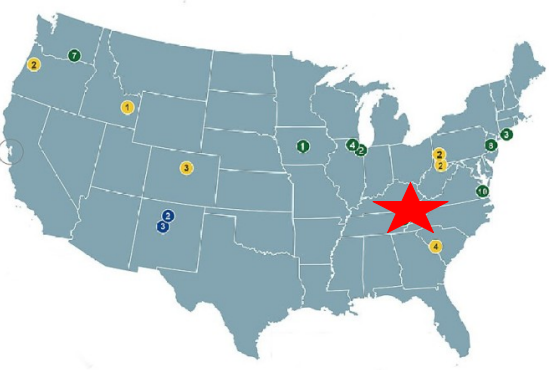

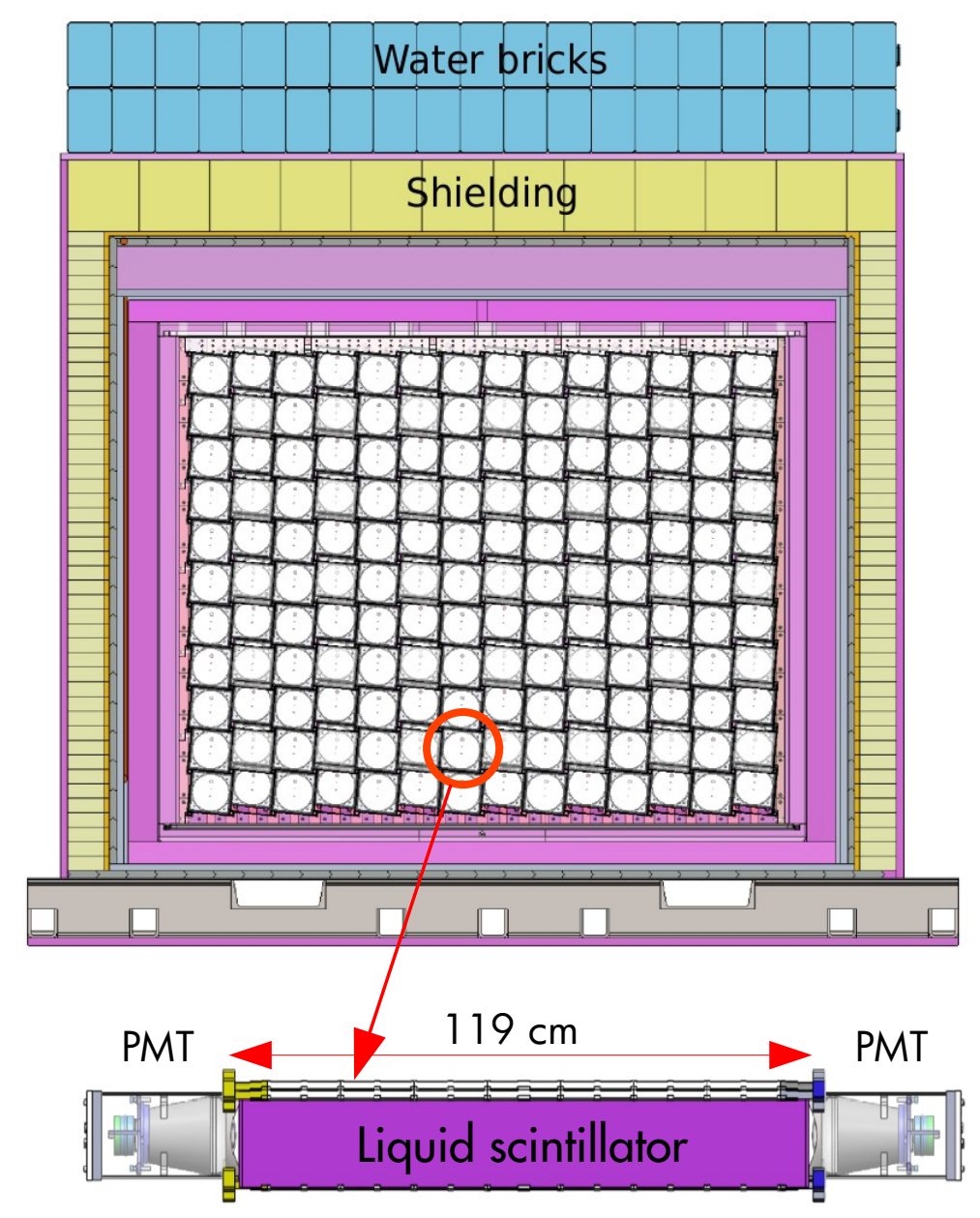




\section{Physical quantities reconstruction}

- The EJ-309 used as base for PROSPECT LiLS has Pulse Shape Discrimination (PSD) capabilities.

- Combine a segment's 2 PMT waveforms into a pulse with reconstructed variables: Energy (electronequivalent), z-position, segment, PSD, ...

- Reconstructed energy is determined by combining charge from both PMTs.

- Heavy particles' energy depositions are modeled using Birks quenching parameters validated with $\mathrm{n}$-Li capture data and fast neutron calibration sources.

J. B. Birks, International series of Monographs on Electronics and Instrumentation, v. 27 Macmillan, New York (1964)
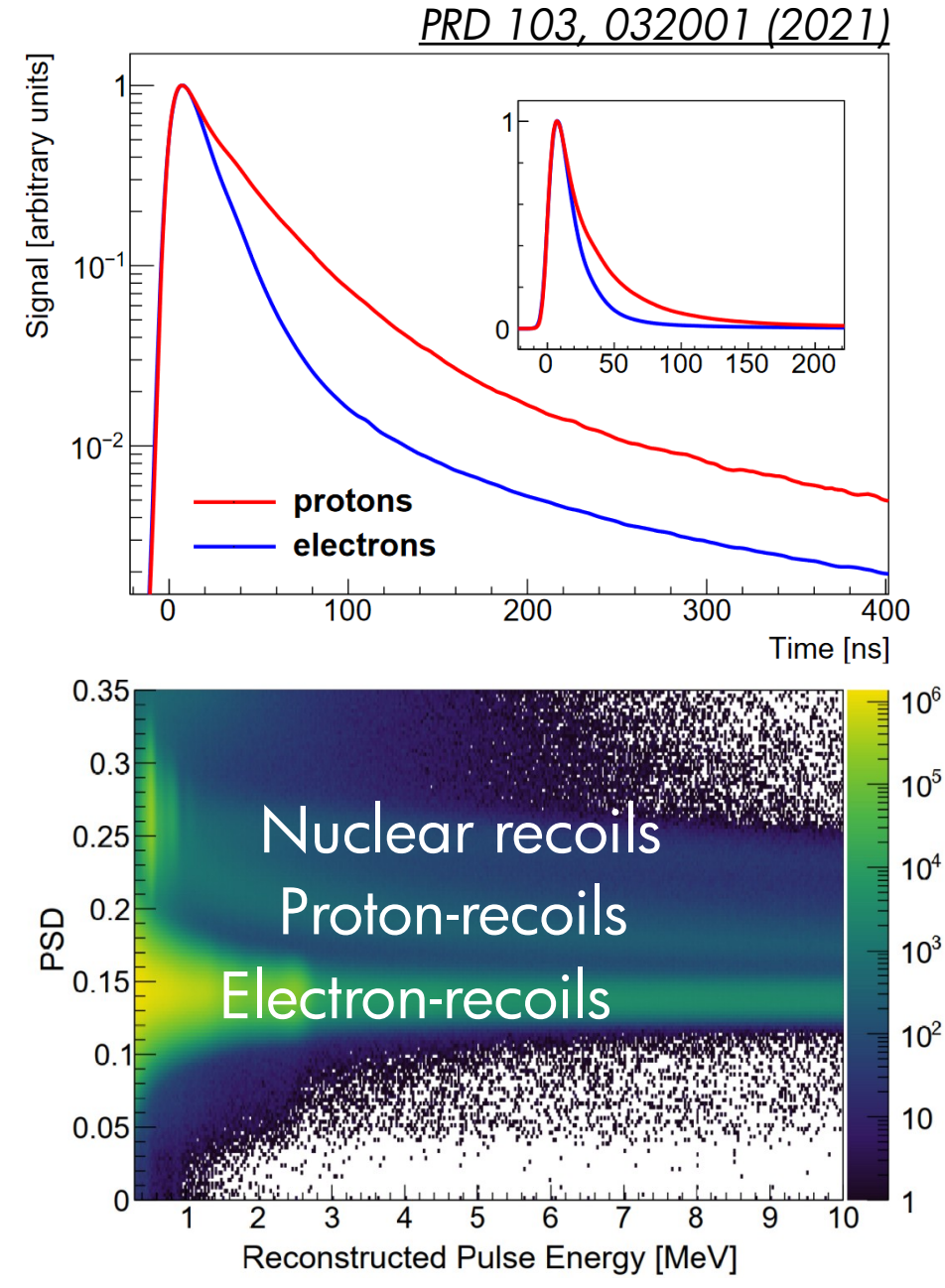


\section{PR@SPECT:}

\section{Signal and Backgrounds}

- Boosted Dark Matters (BDM) scatter with free proton in the LS producing a high PSD single pulse event.

- Detector subjected to multiple backgrounds:

Dark Matter

- PSD rejects environmental gamma backgrounds.

- Single-pulse requirement rejects cosmic $\mathrm{Y}$ M events producing multiple scatters.

- Fiducialization enables detector self-shielding for cosmic neutrons.

- Additional time-based cosmic veto cuts also applied.

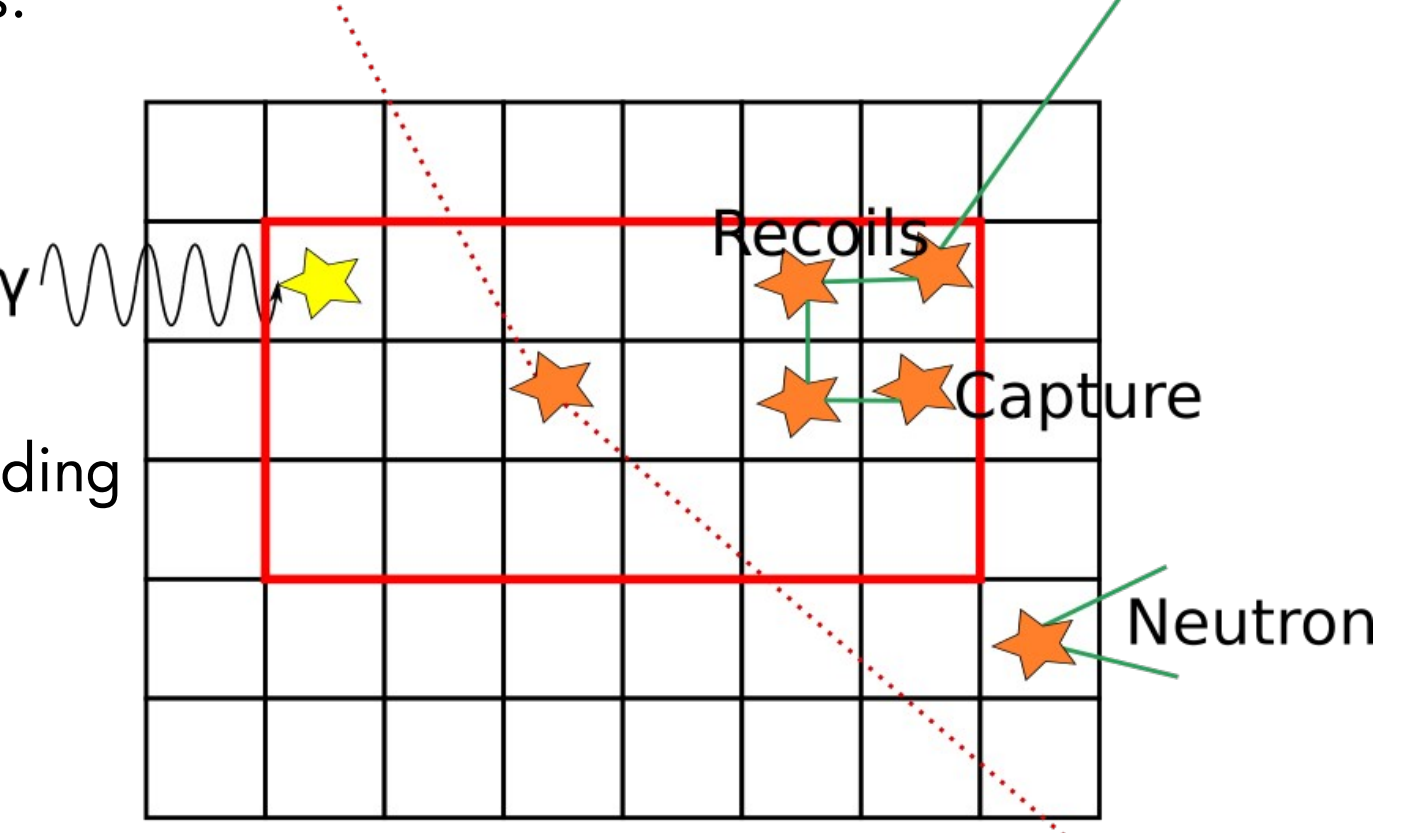




\section{Signal selection}

- 14.6 days of Rx-Off dataset from March $16^{\text {th }} 2018$.

- Set of cuts/vetoes applied to remove backgrounds.

- Background rate decreases by $\sim 2.5-3.5$ order of magnitude.
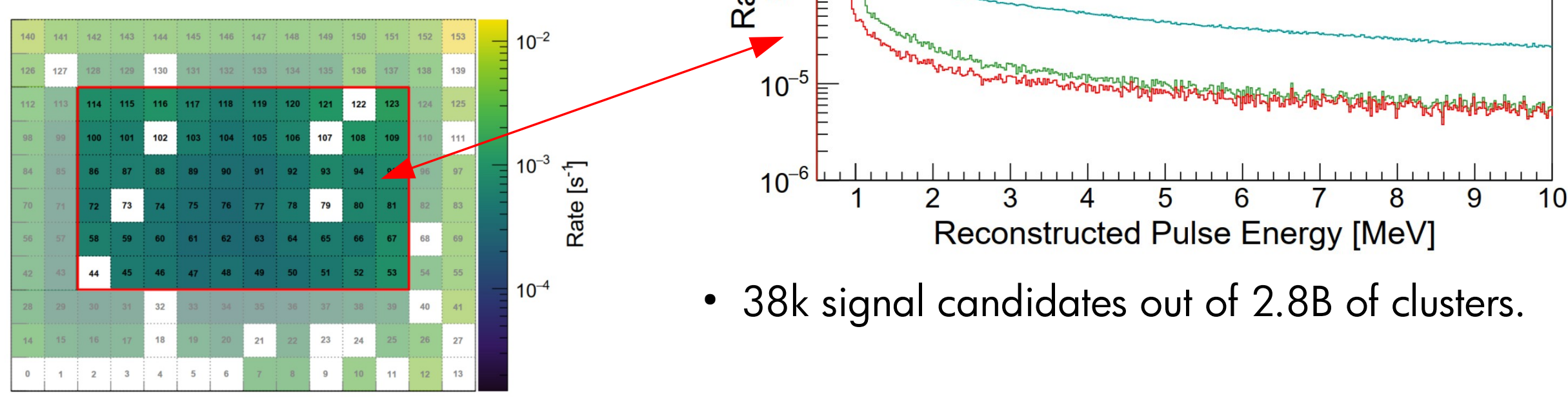

$10^{-4}$

- $38 \mathrm{k}$ signal candidates out of $2.8 \mathrm{~B}$ of clusters. 


\section{Expected vs Measured background variation}

- How do we know cosmogenic backgrounds are not time-varying?

- Neutron capture event rate is $\sim 10 \mathrm{~Hz}$.

- Comparing two time periods of DM flux, - High expected flux: 22:00 - 02:00 GMST - Low expected flux: 10:00 - 14:00 GMST

- Expected level of modulation is: 0.988

- Flat-line fit to ratio shows no modulation: $0.987 \pm 0.003$

- Hourly DM prediction must account for the expected variations in cosmogenic backgrounds.

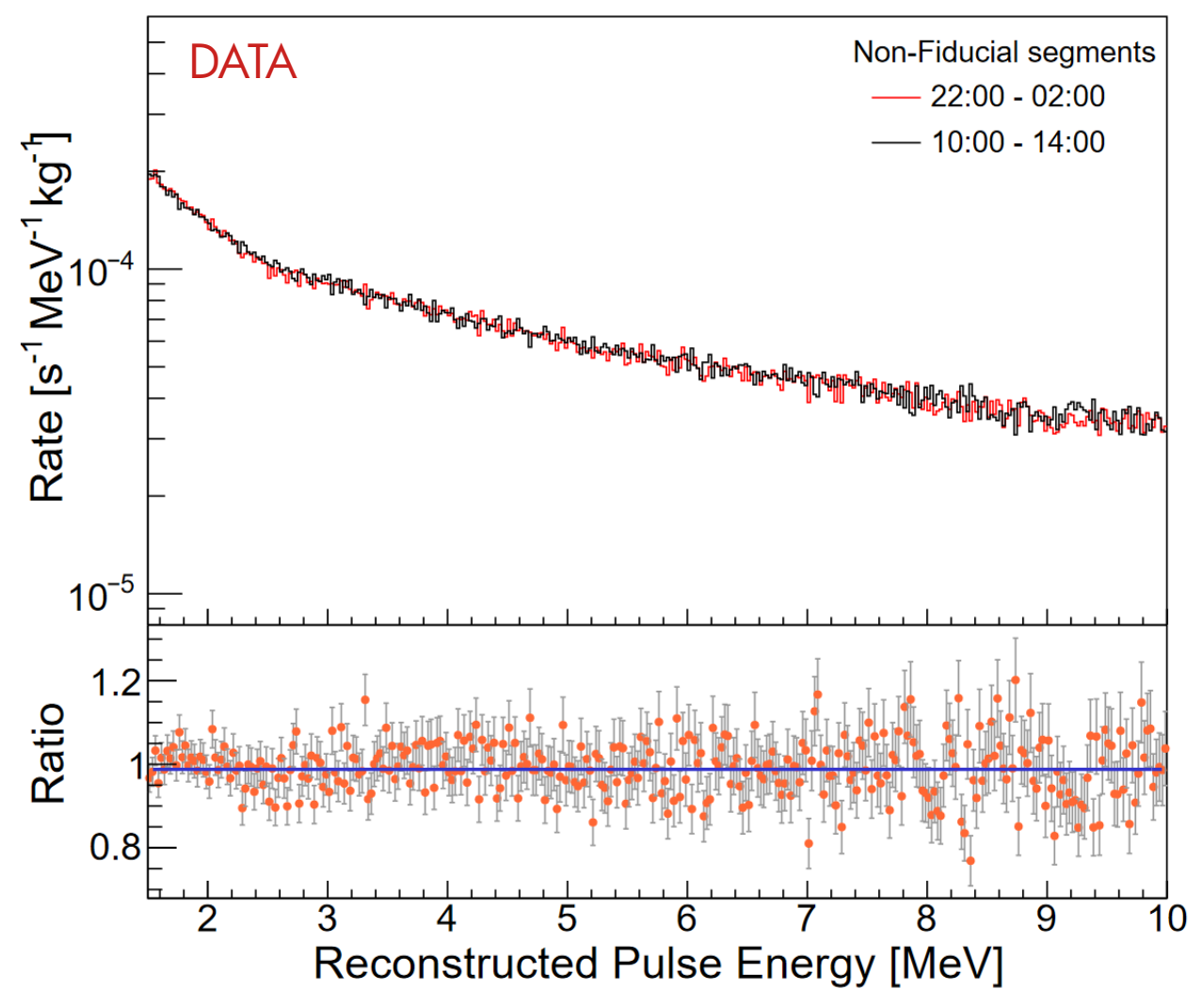




\section{Time modulation of signal}

- How about time modulation of signal?

- Divide the signal spectrum into hour-bin rate.

- No obvious diurnal sidereal modulation.

- Estimate sensitivity/identify excluded space using following chi-square-based test statistic

$$
\Delta \chi^{2}=\chi_{D M}^{2}-\chi_{\text {const }}^{2}
$$

- $\chi_{\text {const }}^{2}$ : flat-line fit to the data.

- $\chi_{D M}^{2}$ : flat-line fit + modulation of $U M\left(m_{x^{\prime}} \sigma_{x N}\right)$ at a time $t$.

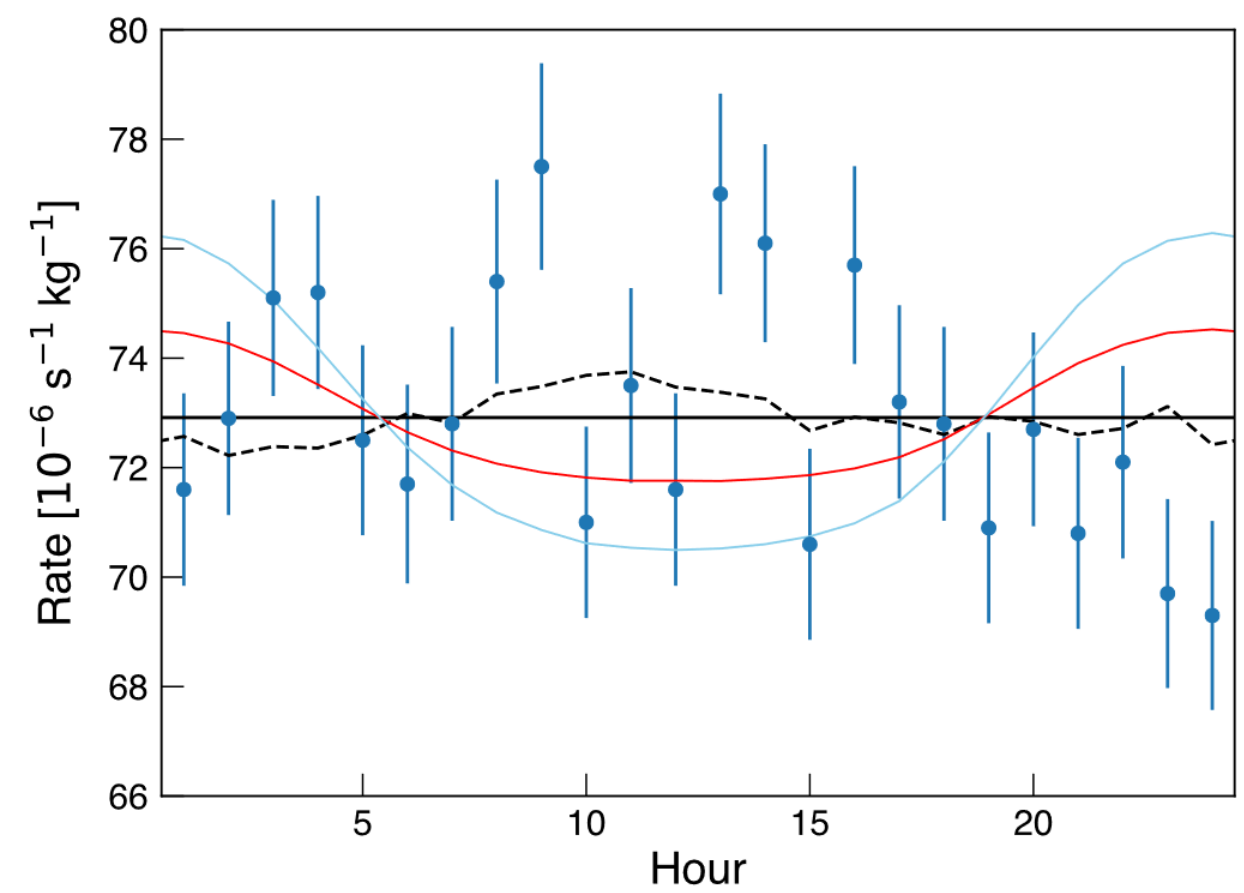




\section{Exclusion region}

- PROSPECT's result covers new space previously un-probed by other terrestrial experiments.

- Upper limit of exclusion is limited by DM attenuation in the atmosphere.

- Lower limit is limited by the fraction of DM interacting with the detector.

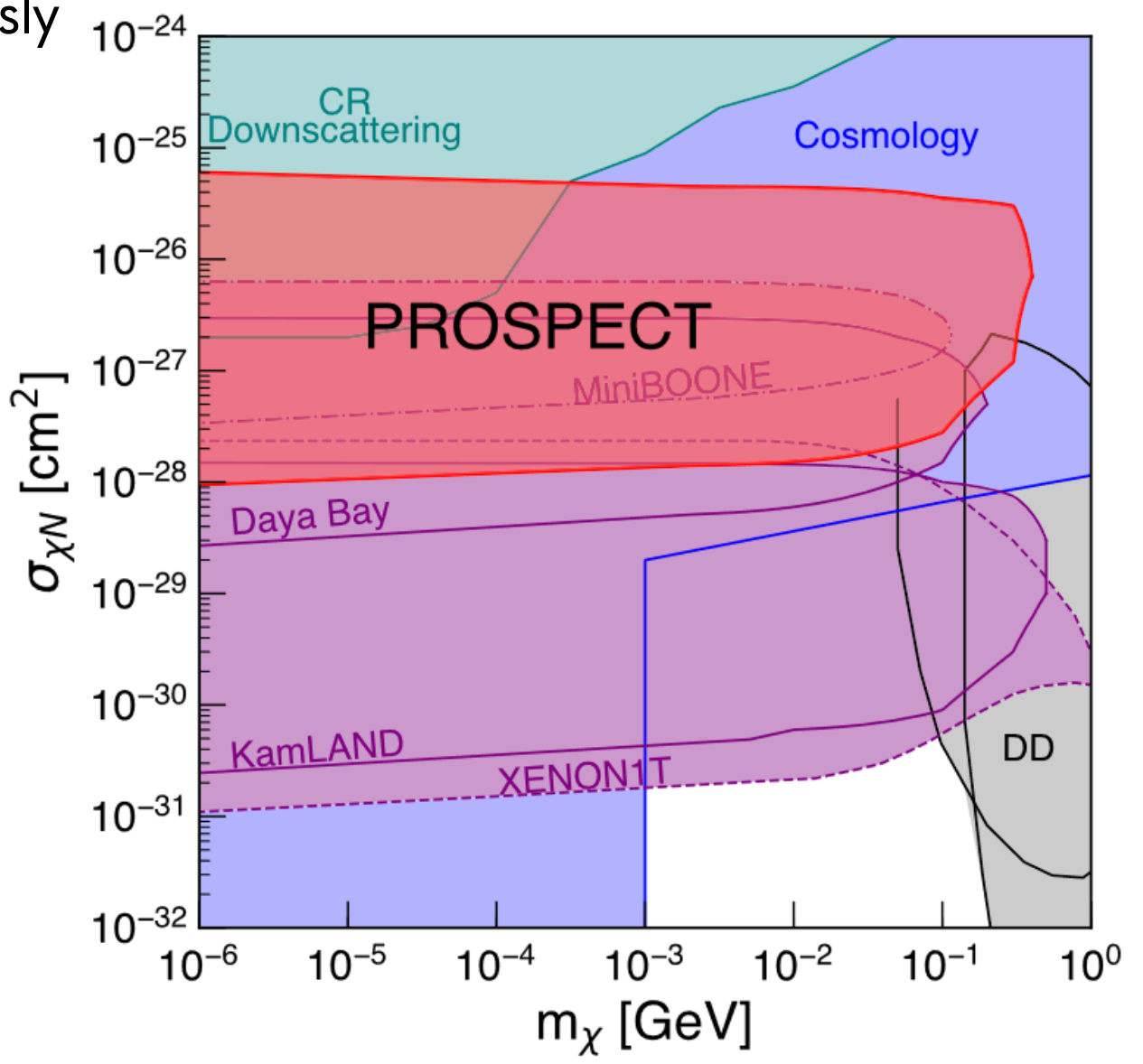




\section{Summary}

- PROSPECT's minimal overburden and events discrimination capabilities allow to probe hard-to-reach Dark Matter phase space.

- In two weeks of data, identified 37,522 candidate events matching the signature of DMproton scattering.

- True presence of a DM signal is expected to produce diurnal modulation in this candidate event rates, while other cosmic backgrounds are expected to produce largely constant event rates

- Our dedicated analysis probes regions of dark matter phase space unaddressed by previous terrestrial experiments.

- Complimentary to cosmological DM limits:

- Other experiments are based on indirect constraint on scattering in the early universe.

- PROPSECT sets limit based on direct detection on scattering in the present day. 
PR@SPEC1 $\bar{\nabla}$ Collaboration Meeting Photo - April 2020

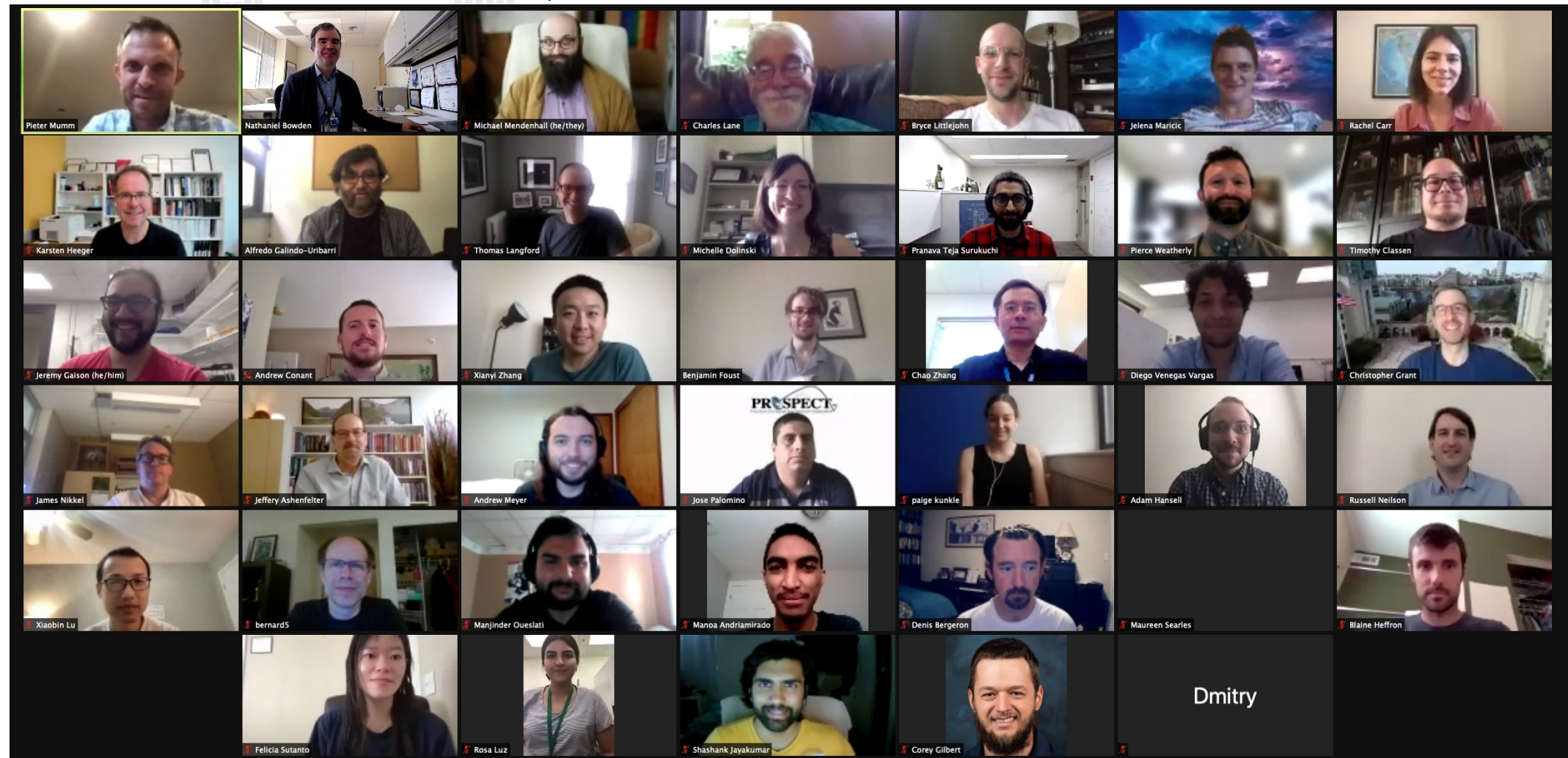

prospect.yale.edu

BRDOKHSWEN

NATIONAL LABORATORY

【Lawrence Livermore

$\triangle$ National Laboratory

\section{OEISING-SIMON}

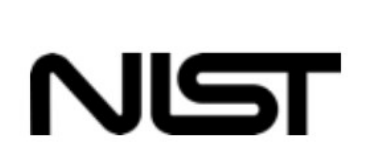

OAK RIDGE National Laboratory

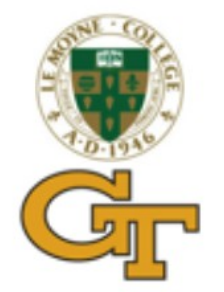

(i5) U.S. DEARETMENT OF Y CRFP
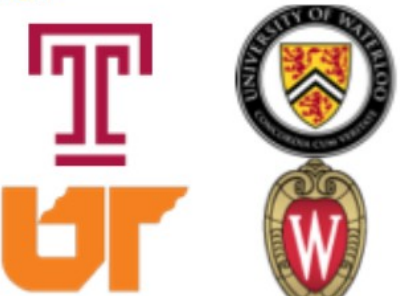

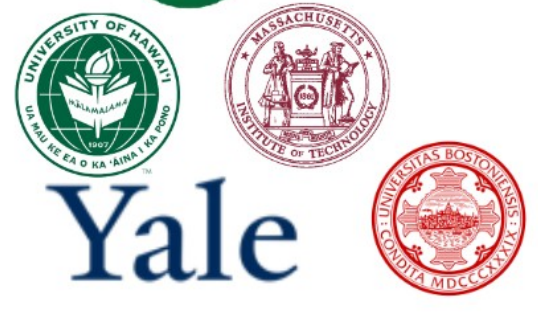

